\title{
A NOVEL METHOD TO IMPROVE NEUROLOGICAL DISEASE SURVEILLANCE
}

Benedict Michael, ${ }^{1}$ Katherine Dodd, ${ }^{2}$ David Geleta, ${ }^{1}$ Terry Payne,

Tom Solomon ${ }^{1}$. 'University of Liverpool; ${ }^{2}$ Countess of Chester Hospital

10.1136/jnnp-2014-309236.41

Introduction Currently, rare neurological diseases are underreported and difficult to recruit to trials and research, in part due to difficulties with current surveillance mechanisms. Smartphones applications are increasingly being used by medical professionals to access up-to-date guidelines, but the potential to use them for data collection has yet to be fully explored.

Method We developed a free smartphone application containing clinical guidelines, which collects data as the doctor reads, and asks readers specific research questions and to notify diseases. The application could also improve recruitment of patients to trials by automatically contacting research teams. 


\section{ABN Abstracts}

Results In the first 6 months the application has been viewed nearly 6000 times to access guidelines, with over 2000 specific disease notifications. Notifications have come from a wide range of countries, specialities, and grades. New cases of TB meningitis and rabies have been reported and also important clinical practice information collected, such as where patients with herpes encephalitis are, and are not, receiving aciclovir within the recommended timeframe.

Conclusion Parallel studies are needed to assess the utility of such applications. Nevertheless, current surveillance mechanisms miss many cases, and this novel approach has the potential to generate real-time disease surveillance data to augment current methods. 